Bounds on Sizes of Finite Bisimulations of Pfaffian Dynamical Systems

Korovina, Margarita and Nicolai, Vorobjov

2008

MIMS EPrint: 2010.4

Manchester Institute for Mathematical Sciences

School of Mathematics

The University of Manchester

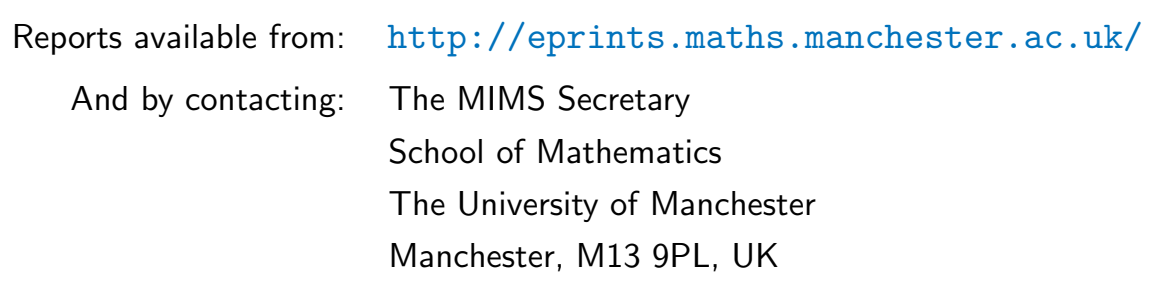

ISSN 1749-9097 


\title{
Bounds on Sizes of Finite Bisimulations of Pfaffian Dynamical Systems
}

\author{
Margarita Korovina • Nicolai Vorobjov
}

Published online: 4 July 2007

(C) Springer Science+Business Media, LLC 2007

\begin{abstract}
We study finite bisimulations of dynamical systems in $\mathbb{R}^{n}$ defined by Pfaffian maps. The pure existence of finite bisimulations for a more general class of ominimal systems was shown in Brihaye et al. (Lecture Notes in Comput. Sci. 2993, 219-233, 2004), Davoren (Theor. Inf. Appl. 33(4/5), 357-382, 1999), Lafferriere et al. (Math. Control Signals Syst. 13, 1-21, 2000). In Lecture Notes in Comput. Sci. 3210, 2004, the authors proved a double exponential upper bound on the size of a bisimulation in terms of the size of description of the dynamical system. In the present paper we improve it to a single exponential upper bound, and show that this bound is tight, by exhibiting a parameterized class of systems on which it is attained.
\end{abstract}

Keywords Dynamical system $\cdot$ Hybrid system $\cdot$ Bisimulation $\cdot$ Semialgebraic geometry

\section{Introduction}

By a Pfaffian dynamical system we mean a map $\gamma: G_{1} \times(-1,1) \rightarrow G_{2}$, where $G_{1} \subset \mathbb{R}^{n-1}$ and $G_{2} \subset \mathbb{R}^{n}$ are open domains and the graph of $\gamma$ is a semi-Pfaffian set (see Definition 2.12). Given a finite partition of $G_{2}$ (consisting of some "areas of interest" which the trajectories have to visit or avoid in a particular order), we aim

M. Korovina $(\bowtie)$

Fachbereich Mathematik, Theoretische Informatik, Universität Siegen, Siegen 57068, Germany

e-mail: korovina@brics.dk

M. Korovina

IIS SB RAS, Novosibirsk, Russia

N. Vorobjov

Department of Computer Science, University of Bath, Bath BA2 7AY, England

e-mail:nnv@cs.bath.ac.uk 
to study a combinatorial dynamical system, having a finite number of trajectories, which is in a natural way isomorphic ("bisimilar") to $\gamma$ with respect to the partition.

A non-constructive existence proof of finite bisimulations for a more general class of o-minimal dynamical systems was given in [2, 3, 11]. The next natural question to investigate is how the sizes of such bisimulations can be bounded. In [8] we gave a double exponential upper bound on the sizes of bisimulations of Pfaffian dynamical systems. We used the cylindrical cell decomposition construction which is intrinsically double exponential. In the present paper we improve that bound to a single exponential upper bound. Moreover we show that the bound is tight, by exhibiting a parameterized class of polynomial dynamical systems on which the exponential bound is attained. The main results of this paper were announced, without proofs, in [9] which also contains a more detailed motivation from the computer science viewpoint, as well as connections with the theory of hybrid systems.

This paper is organized as follows. In Sect. 2 we remind the definitions of transition systems associated to dynamical systems, their bisimulations, Pfaffian functions, semi-Pfaffian sets, and Pfaffian dynamical systems. In Sect. 3 we construct the upper bound on sizes of finite bisimulations of Pfaffian dynamical systems. In Sect. 4 we describe an example of a parameterized class of Pfaffian systems on which the exponential bound is attained. We then conclude with future work.

\section{Basic Notions and Definitions}

\subsection{Transition Systems and Dynamical Systems}

One of the approaches to the study of a dynamical system uses the partition of the state space into finitely many equivalence classes, so that equivalent states exhibit similar properties. This special quotient of the original state space, called bisimulation, is reachability preserving, i.e., checking the reachability on the quotient system is equivalent to checking it on the original system. In this section we recall (following [2]) the notion of bisimulations of transition systems, and basic results concerning finite bisimulations of o-minimal dynamical systems.

The first group of definitions describe transition systems and bisimulations between the transition systems.

Definition 2.1 Let $Q$ be an arbitrary set and $\rightarrow$ be a binary relation on $Q$. In the context of dynamical systems theory we call $Q$ the set of states, $\rightarrow$ the transition, and $T:=(Q, \rightarrow)$ the transition system.

Definition 2.2 Given two transition systems $T_{1}:=\left(Q_{1}, \rightarrow_{1}\right)$ and $T_{2}:=\left(Q_{2}, \rightarrow_{2}\right)$ we define a simulation of $T_{1}$ by $T_{2}$ as a binary relation $\sim \subset Q_{1} \times Q_{2}$ such that:

- $\forall q_{1} \in Q_{1} \exists q_{2} \in Q_{2}\left(q_{1} \sim q_{2}\right)$;

- $\forall q_{1}, q_{1}^{\prime} \in Q_{1} \forall q_{2} \in Q_{2}\left(\left(q_{1} \sim q_{2} \wedge q_{1} \rightarrow_{1} q_{1}^{\prime}\right) \Rightarrow \exists q_{2}^{\prime}\left(q_{1}^{\prime} \sim q_{2}^{\prime} \wedge q_{2} \rightarrow_{2} q_{2}^{\prime}\right)\right)$.

Definition 2.3 A bisimulation between two transition systems $T_{1}:=\left(Q_{1}, \rightarrow_{1}\right)$ and $T_{2}:=\left(Q_{2}, \rightarrow_{2}\right)$ is a simulation $\sim \subset Q_{1} \times Q_{2}$ of $T_{1}$ by $T_{2}$ such that the reciprocal relation $\sim^{-1}:=\left\{\left(q_{2}, q_{1}\right) \in Q_{2} \times Q_{1} \mid q_{1} \sim q_{2}\right\}$ is a simulation of $T_{2}$ by $T_{1}$. 
Definition 2.4 A bisimulation between a transition system $T$ and itself is called bisimulation on $T$.

Definition 2.5 Let $\sim$ be a bisimulation on $T=(Q, \rightarrow)$ and also an equivalence relation on $Q$. Let $\mathcal{P}$ be a partition of $Q$. We say that $\sim$ is a bisimulation with respect to $\mathcal{P}$ if any $P \in \mathcal{P}$ is a union of some equivalence classes of $\sim$.

Normally, the partition $\mathcal{P}$ reflects regions of interest such as invariants and initial conditions of the dynamical system.

In this paper we are concerned with estimating cardinality of bisimulations in the sense of Definition 2.5. We now give some definitions concerning dynamical systems.

Definition 2.6 Let $G_{1} \subset \mathbb{R}^{n-1}$ and $G_{2} \subset \mathbb{R}^{n}$ be open domains. A dynamical system is a map

$$
\gamma: G_{1} \times(-1,1) \rightarrow G_{2}
$$

For a given $\mathbf{x} \in G_{1}$ the set

$$
\Gamma_{\mathbf{x}}=\{\mathbf{y} \mid \exists t \in(-1,1)(\gamma(\mathbf{x}, t)=\mathbf{y})\} \subset G_{2}
$$

is called the trajectory determined by $\mathbf{x}$, and the graph

$$
\widehat{\Gamma}_{\mathbf{x}}=\{(t, \mathbf{y}) \mid \gamma(\mathbf{x}, t)=\mathbf{y}\} \subset(-1,1) \times G_{2}
$$

is called the integral curve determined by $\mathbf{x}$.

A dynamical system is called o-minimal if it is definable in an o-minimal structure over $\mathbb{R}$.

Definition 2.7 The transition system $T_{\gamma}=(Q, \rightarrow)$ associated to the dynamical system $\gamma$ is defined as follows:

- $Q:=G_{2}$, and

- $\mathbf{y}_{1} \rightarrow \mathbf{y}_{2}$ for $\mathbf{y}_{1}, \mathbf{y}_{2} \in Q$ if and only if

$$
\exists \mathbf{x} \in G_{1} \exists t_{1}, t_{2} \in(-1,1)\left(\left(t_{1} \leq t_{2}\right) \wedge\left(\gamma\left(\mathbf{x}, t_{1}\right)=\mathbf{y}_{1}\right) \wedge\left(\gamma\left(\mathbf{x}, t_{2}\right)=\mathbf{y}_{2}\right)\right) .
$$

We now introduce following [2], a technique of encoding trajectories of dynamical systems by words. Let $\mathcal{P}:=\left\{P_{1}, \ldots, P_{s}\right\}$ be a finite partition of $\gamma\left(G_{1} \times(-1,1)\right)$ definable in the o-minimal structure. Fix $\mathbf{x} \in G_{1}$. Define the set $\mathcal{F}_{\mathbf{x}}$ of points and open intervals $I$ in $(-1,1)$ which are maximal with respect to inclusion for the property

$$
\exists i \in\{1, \ldots, s\} \quad \forall t \in I\left(\gamma(\mathbf{x}, t) \in P_{i}\right) .
$$

Let the cardinality $\left|\mathcal{F}_{\mathbf{x}}\right|=r$ and $y_{1}<\cdots<y_{r}$ be the set of representatives of $\mathcal{F}_{\mathbf{x}}$ such that $\gamma\left(\mathbf{x}, y_{j}\right) \in P_{i_{j}}$. Then define the word $\omega:=P_{i_{1}} \cdots P_{i_{r}}$ in the alphabet $\mathcal{P}$. Informally, $\omega$ is the list of names of elements of the partition in the order they are visited by the trajectory $\Gamma_{\mathbf{x}}$. 
Let $\mathbf{y} \in \Gamma_{\mathbf{x}}$. Then $\mathbf{y} \in P_{i_{j}}$ for some $1 \leq j \leq r$, where $P_{i_{j}}$ is a letter in $\omega$. We represent the location of $\mathbf{y}$ on trajectory $\Gamma_{\mathbf{x}}$ by the dotted word

$$
\dot{\omega}:=P_{i_{1}} \cdots \dot{P}_{i_{j}} \cdots P_{i_{r}}
$$

In the sequel we will always assume that the dynamical system $\gamma$ is injective. In this case there is a unique dotted word associated to a given $\mathbf{y} \in \gamma\left(G_{1} \times(-1,1)\right)$. Introduce sets of words $\Omega:=\left\{\omega \mid \mathbf{x} \in G_{1}\right\}, \dot{\Omega}:=\left\{\dot{\omega} \mid \mathbf{x} \in G_{1}\right\}$. The following statement is an easy consequence of o-minimality.

Lemma 2.8 ([2]) The set $\Omega$ is finite.

An obvious (purely combinatorial) corollary is that $\dot{\Omega}$ is also finite.

Definition 2.9 The transition system $T_{\dot{\Omega}}$ is defined as follows:

- $Q:=\dot{\Omega}$, and

- $\dot{\omega}_{1} \rightarrow \dot{\omega}_{2}$ for $\dot{\omega}_{1}, \dot{\omega}_{2} \in Q$ if and only if $\omega_{1}=\omega_{2}$ and the dot on $\dot{\omega}_{2}$ is on the righter (or the same) position than the dot on $\dot{\omega}_{1}$.

Theorem 2.10 ([2]) Let an o-minimal dynamical system $\gamma$ be bijective, and the partition $\mathcal{P}$ be definable in the o-minimal structure. Then there is a finite bisimulation on $T_{\gamma}$ with respect to $\mathcal{P}$.

Proof To prove the theorem one first shows that $T_{\dot{\Omega}}$ is a bisimulation of $T_{\gamma}$, and then considers the following equivalence relation $\sim$ on $G_{2}: \mathbf{y}_{1} \sim \mathbf{y}_{2}$ iff for respective preimages $\left(\mathbf{x}_{1}, t_{1}\right),\left(\mathbf{x}_{2}, t_{2}\right)$, the locations of $\mathbf{y}_{1}, \mathbf{y}_{2}$ on trajectories $\Gamma_{\mathbf{x}_{1}}, \Gamma_{\mathbf{x}_{2}}$ are described by the same dotted word $\dot{\omega}$. Then $\sim$ is the required bisimulation (see details in [2]).

\subsection{Pfaffian Functions and Related Sets}

In what follows, in order to give a quantitative refinement of Theorem 2.10 we will restrict our considerations of o-minimal dynamical systems to a particular case, the class of Pfaffian dynamical systems. This section is a digest of the theory of Pfaffian functions and sets definable with Pfaffian functions. The detailed exposition can be found in the survey [5].

Definition 2.11 A Pfaffian chain of order $r \geq 0$ and degree $\alpha \geq 1$ in an open domain $G \subset \mathbb{R}^{n}$ is a sequence of real analytic functions $f_{1}, \ldots, f_{r}$ in $G$ satisfying differential equations

$$
\frac{\partial f_{j}}{\partial x_{i}}=g_{i j}\left(\mathbf{x}, f_{1}(\mathbf{x}), \ldots, f_{j}(\mathbf{x})\right)
$$

for $1 \leq j \leq r, 1 \leq i \leq n$. Here $g_{i j}\left(\mathbf{x}, y_{1}, \ldots, y_{j}\right)$ are polynomials in $\mathbf{x}=\left(x_{1}, \ldots, x_{n}\right)$, $y_{1}, \ldots, y_{j}$ of degrees not exceeding $\alpha$. A function

$$
f(\mathbf{x})=P\left(\mathbf{x}, f_{1}(\mathbf{x}), \ldots, f_{r}(\mathbf{x})\right),
$$


where $P\left(\mathbf{x}, y_{1}, \ldots, y_{r}\right)$ is a polynomial of a degree not exceeding $\beta \geq 1$, is called a Pfaffian function of order $r$ and degree $(\alpha, \beta)$.

In order to illustrate the definition let us consider several examples of Pfaffian functions.

(a) Pfaffian functions of order 0 and degree $(1, \beta)$ are polynomials of degrees not exceeding $\beta$.

(b) The exponential function $f(x)=e^{a x}$ is a Pfaffian function of order 1 and degree $(1,1)$ in $\mathbb{R}$, due to the equation $d f(x)=a f(x) d x$. More generally, for $i=1,2, \ldots, r$, let $E_{i}(x):=e^{E_{i-1}(x)}, E_{0}(x)=a x$. Then $E_{r}(x)$ is a Pfaffian function of order $r$ and degree $(r, 1)$, since $d E_{r}(x)=a E_{1}(x) \cdots E_{r}(x) d x$.

(c) The function $f(x)=1 / x$ is a Pfaffian function of order 1 and degree $(2,1)$ in the domain $\{x \in \mathbb{R} \mid x \neq 0\}$, due to the equation $d f(x)=-f^{2}(x) d x$.

(d) The logarithmic function $f(x)=\ln (|x|)$ is a Pfaffian function of order 2 and degree $(2,1)$ in the domain $\{x \in \mathbb{R} \mid x \neq 0\}$, due to equations $d f(x)=g(x) d x$ and $d g(x)=-g^{2}(x) d x$, where $g(x)=1 / x$.

(e) The polynomial $f(x)=x^{m}$ can be viewed as a Pfaffian function of order 2 and degree $(2,1)$ in the domain $\{x \in \mathbb{R} \mid x \neq 0\}$ (but not in $\mathbb{R}$ ), due to the equations $d f(x)=m f(x) g(x) d x$ and $d g(x)=-g^{2}(x) d x$, where $g(x)=1 / x$. In some cases a better way to deal with $x^{m}$ is to change the variable $x=e^{u}$ reducing this case to (b).

(f) The function $f(x)=\tan (x)$ is a Pfaffian function of order 1 and degree $(2,1)$ in the domain $\bigcap_{k \in \mathbb{Z}}\{x \in \mathbb{R} \mid x \neq \pi / 2+k \pi\}$, due to the equation $d f(x)=$ $\left(1+f^{2}(x)\right) d x$.

(g) The function $f(x)=\arctan (x)$ is a Pfaffian function in $\mathbb{R}$ of order 2 and degree $(3,1)$, due to equations $d f(x)=g(x) d x$ and $d g(x)=-2 x g^{2}(x) d x$, where $g(x)=\left(x^{2}+1\right)^{-1}$.

(h) The function $\cos (x)$ is a Pfaffian function of order 2 and degree $(2,1)$ in the domain $\bigcap_{k \in \mathbb{Z}}\{x \in \mathbb{R} \mid x \neq \pi+2 k \pi\}$, due to equations $\cos (x)=2 f(x)-1, d f(x)=$ $-f(x) g(x) d x$, and $d g(x)=\frac{1}{2}\left(1+g^{2}(x)\right) d x$, where $f(x)=\cos ^{2}(x / 2)$ and $g(x)=\tan (x / 2)$. Also, since $\cos (x)$ is a polynomial of degree $m$ of $\cos (x / m)$, the function $\cos (x)$ is Pfaffian of order 2 and degree $(2, m)$ in the domain $\bigcap_{k \in \mathbb{Z}}\{x \in \mathbb{R} \mid x \neq m \pi+2 k m \pi\}$. The same is true, of course, for any shift of this domain by a multiple of $\pi$. However, $\cos (x)$ is not a Pfaffian function in the whole real line.

As we can see, apart from polynomials, the class of Pfaffian functions includes real algebraic functions, exponentials, logarithms, trigonometric functions, their compositions, and other major transcendental functions in appropriate domains (see [5]). Now we introduce classes of sets definable with Pfaffian functions. In the case of polynomials they reduce to semialgebraic sets whose quantitative and algorithmic theory is treated in [1].

Definition 2.12 A set $X \subset \mathbb{R}^{n}$ is called semi-Pfaffian in an open domain $G \subset \mathbb{R}^{n}$ if it consists of points in $G$ satisfying a Boolean combination of some atomic equations and inequalities $f=0, g>0$, where $f, g$ are Pfaffian functions having a common 
Pfaffian chain defined in $G$. A semi-Pfaffian set $X$ is restricted in $G$ if its topological closure lies in $G$.

Definition 2.13 A set $X \subset \mathbb{R}^{n}$ is called sub-Pfaffian in an open domain $G \subset \mathbb{R}^{n}$ if it is an image of semi-Pfaffian set under a projection into a subspace.

In the sequel we will be dealing with the following subclass of sub-Pfaffian sets.

Definition 2.14 Consider the closed cube $[-1,1]^{m+n}$ in an open domain $G \subset \mathbb{R}^{m+n}$ and the projection map $\pi: \mathbb{R}^{m+n} \rightarrow \mathbb{R}^{n}$. A subset $Y \subset[-1,1]^{n}$ is called restricted sub-Pfaffian if $Y=\pi(X)$ for a restricted semi-Pfaffian set $X \subset[-1,1]^{m+n}$.

Note that a restricted sub-Pfaffian set need not be semi-Pfaffian.

Definition 2.15 Consider a semi-Pfaffian set

$$
X:=\bigcup_{1 \leq i \leq M}\left\{\mathbf{x} \in \mathbb{R}^{s} \mid f_{i 1}=0, \ldots, f_{i I_{i}}=0, g_{i 1}>0, \ldots, g_{i J_{i}}>0\right\} \subset G,
$$

where $f_{i j}, g_{i j}$ are Pfaffian functions with a common Pfaffian chain of order $r$ and degree $(\alpha, \beta)$, defined in an open domain $G$. Its format is a tuple $(r, N, \alpha, \beta, s)$, where $N \geq \sum_{1 \leq i \leq M}\left(I_{i}+J_{i}\right)$. If $Y \subset \mathbb{R}^{n}$ is a sub-Pfaffian subset such that $Y=\pi(X)$ and $s=m+n$, then its format is equal to the format of $X$.

We will refer to the representation of a semi-Pfaffian set in the form (2.2) as to disjunctive normal form (DNF).

Remark 2.16 In this paper we are concerned with upper and lower bounds on sizes of bisimulations as functions of the format. In the case of Pfaffian dynamical systems these sizes and complexities also depend on the domain $G$. So far our definitions have imposed no restrictions on the open set $G$, thus allowing it to be arbitrarily complex and induce this complexity on the corresponding semi- and sub-Pfaffian sets. To avoid this we will always assume in the context of Pfaffian dynamical systems that $G$ is simple, like $\mathbb{R}^{n}$, or $(-1,1)^{n}$.

Theorem 2.17 ([5, 13]) Consider a semi-Pfaffian set $X \subset G \subset \mathbb{R}^{n}$, where $G$ is an open domain, represented in DNF with format $(r, N, \alpha, \beta, n)$. Then the sum of the Betti numbers (in particular, the number of connected components) of $X$ does not exceed

$$
N^{n} 2^{r(r-1) / 2} O(n \beta+\min \{n, r\} \alpha)^{n+r} .
$$

Theorem 2.18 ([6, Sect. 5.2]) Consider a sub-Pfaffian set $Y=\pi(X)$ as described in Definition 2.14. Let $X$ be closed and represented in DNF with format $(r, N, \alpha, \beta$, $n+m)$. Then the kth Betti number $\mathrm{b}_{k}(Y)$ does not exceed

$$
\begin{aligned}
& k((k+1) N)^{n+(k+1) m} 2^{(k+1) r((k+1) r-1) / 2} \cdot O((n+k m) \beta \\
& \quad+\min \{k r, n+k m\} \alpha)^{n+(k+1)(m+r)} .
\end{aligned}
$$


Let $d>\alpha+\beta$. Relaxing the bound from Theorem 2.18, we get

$$
\mathrm{b}_{k}(Y) \leq(k N)^{O(n+k m)} 2^{O\left((k r)^{2}\right)}((n+k m) d)^{O(n+k m+k r)} .
$$

\subsection{Singular Loci of Semi-Pfaffian Sets}

Consider a semi-Pfaffian set

$$
X:=\bigcup_{1 \leq i \leq M}\left\{\mathbf{x} \in \mathbb{R}^{n} \mid f_{i 1}=0, \ldots, f_{i I_{i}}=0, g_{i 1}>0, \ldots, g_{i J_{i}}>0\right\} \subset G,
$$

where $f_{i j}, g_{i j}$ are Pfaffian functions with a common Pfaffian chain, defined in an open domain $G$. Let the format of $X$ be $(r, N, \alpha, \beta, n)$, where $N \geq \sum_{1 \leq i \leq M}\left(I_{i}+J_{i}\right)$.

Assume that $X$ is a $p$-dimensional topological (not necessarily smooth) manifold. We say that $\mathbf{x}$ is smooth if in the neighbourhood of $\mathbf{x}$ the set $X$ is a $C^{1}$-manifold, and singular otherwise. Let $\mathcal{S}_{\mathbf{x}}$ be a secant cone at a point $\mathbf{x} \in X$, i.e., the limit of all secant straight lines through pairs of points in $X$ converging to $\mathbf{x}$.

Theorem 2.19 A point $\mathbf{x} \in X$ is singular if and only if the dimension of the affine hull of $S_{\mathbf{x}}$ is larger than $p$.

Lemma 2.20 $S_{\mathbf{x}}$ is an affine space of dimension $p$ if and only if $\mathbf{x}$ is smooth.

Proof If $\mathbf{x}$ is smooth, then $S_{\mathbf{x}}$ coincides with the tangent space to $X$ at $\mathbf{x}$, and therefore is a $p$-dimensional affine space.

Suppose now that $S_{\mathbf{X}}$ is an affine space of the dimension $p$. Let $f: X \rightarrow S_{\mathbf{X}}$ be the orthogonal projection. For a neighbourhood $U_{\mathbf{x}}$ of $\mathbf{x}$ in $X$ (which is homeomorphic to an open ball) the restriction $\left.f\right|_{U_{\mathbf{x}}}$ is injective. Indeed, suppose that for any neighbourhood $U_{\mathbf{x}}$ there exist $\mathbf{x}_{1}, \mathbf{x}_{2} \in U_{\mathbf{x}}$ such that $\mathbf{x}_{1} \neq \mathbf{x}_{2}$ and $f\left(\mathbf{x}_{1}\right)=f\left(\mathbf{x}_{2}\right)$. Then, the line through $\mathbf{x}_{1}, \mathbf{x}_{2}$ is orthogonal to $S_{\mathbf{x}}$ which contradicts the fact that its limit is contained in $S_{\mathbf{x}}$. It follows that $\left.f\right|_{U_{\mathbf{x}}}$ is a homeomorphism of open balls.

Now let $L_{\mathbf{x}}$ be the $(n-p)$-dimensional affine subspace passing through $\mathbf{x}$ and orthogonal to $S_{\mathbf{x}}$. Consider the map $F=\left(F_{1}, \ldots, F_{n-p}\right): f\left(U_{\mathbf{x}}\right) \rightarrow L_{\mathbf{x}}$ for which $U_{\mathbf{x}}$ is the graph. Then each $F_{i}$ is $C^{1}$-differentiable by the definition (all partial derivatives of $F_{i}$ are 0 at $\mathbf{x}$ ). Hence $U_{\mathbf{x}}$ is smooth as the graph of a smooth map.

Lemma 2.21 For any $\mathbf{x} \in X$ the secant cone $S_{\mathbf{x}}$ contains a $p$-dimensional affine space.

Proof Let $R_{\mathbf{X}}$ be the union of all limits of tangent spaces to smooth points converging to $\mathbf{x}$. Clearly, $R_{\mathbf{x}}$ contains a $p$-dimensional affine space. On the other hand, $R_{\mathbf{x}} \subset S_{\mathbf{x}}$.

Proof of Theorem 2.19 By Lemmas 2.20, 2.21, $\mathbf{x} \in X$ is singular if and only if $S_{\mathbf{x}}$ contains a $p$-dimensional affine space but not coincides with it. 
Theorem 2.22 The set $X_{\text {sing }}$ of all singular points of $X$ is sub-Pfaffian, representable by an existential formula with the format $\left(r,\left(N^{O(n)} D\right)^{n+r}, \alpha, D, O\left(n^{2}\right)\right)$, where

$$
D:=2^{O\left(n r^{2}\right)}(n(\alpha+\beta))^{O(n(n+r))} .
$$

Proof Define the secant bundle of $X$ as

$$
\mathcal{S} X:=\left\{(\mathbf{x}, \mathbf{v}) \in X \times \mathbb{R}^{n} \mid(\mathbf{x}, \mathbf{x}, \mathbf{v}) \in \operatorname{closure}(\mathcal{S})\right\},
$$

where

$$
\mathcal{S}:=\left\{(\mathbf{x}, \mathbf{y}, \lambda(\mathbf{y}-\mathbf{x})) \in X \times X \times \mathbb{R}^{n} \mid((\mathbf{x} \neq \mathbf{y}) \wedge(\lambda \in \mathbb{R}))\right\}
$$

is the set of all triples $(\mathbf{x}, \mathbf{y}, \mathbf{v})$ in which $\mathbf{v}$ is a vector parallel to the line joining two distinct points $\mathbf{x}, \mathbf{y} \in X$. A theorem of Gabrielov ([5, Theorem 5.2]) implies that the set $\mathcal{S} X$ is semi-Pfaffian, and its format can be bounded explicitly via the format of $X$. According to Theorem 2.19, a point $\mathbf{x}_{0} \in X$ is singular if and only if the affine hull of the secant cone

$$
\mathcal{S}_{\mathbf{x}_{0}}:=\left\{\mathbf{v} \in \mathbb{R}^{n} \mid\left(\mathbf{x}_{0}, \mathbf{x}_{0}, \mathbf{v}\right) \in \operatorname{closure}(\mathcal{S})\right\},
$$

at $\mathbf{x}_{0}$ has the dimension larger than $\operatorname{dim}(X)=p$. Thus,

$$
\begin{aligned}
& X_{\text {sing }}=\left\{\mathbf{x} \in X \mid \exists\left(\mathbf{y}_{1}, \ldots, \mathbf{y}_{p+1}\right)\left(\mathbf{y}_{1} \in \mathcal{S}_{\mathbf{x}} \wedge \cdots\right.\right. \\
&\left.\left.\cdots \wedge \mathbf{y}_{p+1} \in \mathcal{S}_{\mathbf{x}} \wedge \operatorname{rank}\left(\mathbf{y}_{1} \cdots \mathbf{y}_{p+1}\right)=p+1\right)\right\} .
\end{aligned}
$$

A straightforward estimate of the format of this formula completes the proof.

\section{The Upper Bound on Sizes of Finite Bisimulation of Pfaffian Dynamical Systems}

Our main results concern upper and lower bounds for finite bisimulations of Pfaffian dynamical systems with respect to partitions defined by semi-Pfaffian sets.

Definition 3.1 A dynamical system

$$
\gamma: G_{1} \times(-1,1) \rightarrow G_{2},
$$

where $G_{1} \subseteq \mathbb{R}^{n-1}$ and $G_{2} \subseteq \mathbb{R}^{n}$ are open and $\gamma$ is a map with a semi-Pfaffian graph, is called a Pfaffian dynamical system.

Let $\gamma: G_{1} \times(-1,1) \rightarrow G_{2}$, where $G_{1}=I^{n-1}:=(-1,1)^{n-1}$ and $G_{2}=I^{n}$, be a homeomorphism, defined by its graph

$$
\widehat{\Gamma}:=\{(\mathbf{x}, t, \mathbf{y}) \mid \gamma(\mathbf{x}, t)=\mathbf{y}\}
$$

which is a semi-Pfaffian set, and $\mathcal{P}$ be a partition of $G_{2}$ into semi-Pfaffian sets.

First we consider an elementary example illustrating techniques which we will use to show the single exponential upper bounds in the general case. 


\subsection{Example}

Let $G_{1}:=(-1,1), G_{2}:=(-1,1)^{2}$, and

$$
\gamma:(x, t) \mapsto\left(y_{1}=x, y_{2}=t\right) .
$$

(Note that this dynamical system corresponds to the system of differential equations $\dot{y}_{1}=0, \dot{y}_{2}=1$.) Consider the graph

$$
\widehat{\Gamma}:=\left\{\left(x, t, y_{1}, y_{2}\right) \mid x-y_{1}=0, t-y_{2}=0\right\}
$$

of the map $\gamma$. Note that $\widehat{\Gamma}$ is an intersection of 4-cube $(-1,1)^{4}$ with a 2-plane, and therefore is a smooth manifold. In the general case the graph of a dynamical system may not be smooth and we will need to separate smooth and singular parts of it. For a fixed $x \in G_{1}$ the set

$$
\widehat{\Gamma}_{x}:=\left\{\left(t, y_{1}, y_{2}\right) \mid x-y_{1}=0, t-y_{2}=0\right\}
$$

is the integral curve, and the set

$$
\Gamma_{x}:=\left\{\left(y_{1}, y_{2}\right) \mid \exists t\left(x-y_{1}=0, t-y_{2}=0\right)\right\}
$$

is the trajectory of $\gamma$. Thus, in our example, the trajectories are open segments of straight lines parallel to $y_{2}$-axis.

Introduce the projection

$$
\pi:\left\{\begin{array}{l}
G_{1} \times(-1,1) \times G_{2} \rightarrow G_{1} \\
\left(x, t, y_{1}, y_{2}\right) \mapsto x .
\end{array}\right.
$$

Let $\pi \widehat{\Gamma}$ be the restriction of $\pi$ to $\widehat{\Gamma}$. For a fixed $x \in G_{1}$ the fiber $\pi_{\widehat{\Gamma}}^{-1}(x)$ coincides with $\widehat{\Gamma}_{x}$.

Let the partition $\mathcal{P}$ of $G_{2}$ consist of the $\operatorname{disc}\left\{\left(y_{1}, y_{2}\right) \mid f:=y_{1}^{2}+y_{2}^{2}-1 / 4 \leq 0\right\}$ labelled by the letter $A$ and its complement in $G_{2}$ labelled by $B$. The aim is to determine the number of different words in the alphabet $\{A, B\}$ encoding the trajectories. Clearly, it is sufficient to consider only intersections of the trajectories with open sets $\left\{\left(y_{1}, y_{2}\right) \mid f<0\right\}$ and $\left\{\left(y_{1}, y_{2}\right) \mid f>0\right\}$ (in the general case, the transition to open sets is less trivial and the subject of Subsect. 3.3).

Let $\widehat{S}:=\left\{\left(x, t, y_{1}, y_{2}\right) \mid f\left(y_{1}, y_{2}\right)=0\right\}$. Observe that $\widehat{S} \cap \widehat{\Gamma}$ is a smooth curve. Consider the partition $\widehat{\mathcal{P}}$ of $\widehat{\Gamma}$ consisting of $\left\{\left(x, t, y_{1}, y_{2}\right) \mid f:=y_{1}^{2}+y_{2}^{2}-1 / 4 \leq 0\right\}$ labelled by letter $A$ and its complement in $\widehat{\Gamma}$ labelled by $B$. Clearly, it is sufficient to find the number of distinct words encoding the intersections of integral curves with open sets $\left\{\left(x, t, y_{1}, y_{2}\right) \mid f<0\right\} \cap \widehat{\Gamma}$ and $\left\{\left(x, t, y_{1}, y_{2}\right) \mid f>0\right\} \cap \widehat{\Gamma}$.

Consider the restriction $\pi \widehat{\Gamma} \widehat{S}: \widehat{\Gamma} \rightarrow G_{1}$ of $\pi \widehat{\Gamma}$ to $\widehat{S} \cap \widehat{\Gamma}$. Let $C$ be the set of all critical values of $\pi \widehat{\Gamma} \widehat{S}$. By setting to 0 the appropriate Jacobian we find that the critical points of $\pi \widehat{\Gamma} \widehat{S}$ are $(1 / 2,0,1 / 2,0)$ and $(-1 / 2,0,-1 / 2,0)$, thus $C=\{1 / 2,-1 / 2\}$. 
Let $R:=G_{1} \backslash C$. This set consists of three connected components:

$$
\begin{aligned}
& \{x \in(-1,1) \mid x<-1 / 2\}, \\
& \{x \in(-1,1) \mid-1 / 2<x<1 / 2\}, \\
& \{x \in(-1,1) \mid 1 / 2<x\} .
\end{aligned}
$$

The following statement is obvious.

Proposition 3.2 If $x, x^{\prime}$ belong to the same connected component $R^{\prime}$ of $R$, then $\widehat{\Gamma}_{x}$ and $\widehat{\Gamma}_{x^{\prime}}$ are labelled by the same word.

In the general case the analogy of Proposition 3.2 requires a careful proof. As applied to our example, this proof has the following scheme.

(1) The restriction of $\pi_{\widehat{\Gamma} \widehat{S}}$ to $\pi_{\widehat{\Gamma} \widehat{S}}^{-1}\left(R^{\prime}\right)$ is a trivial covering, i.e., for any $x^{\prime} \in R^{\prime}$ the pre-image $\pi_{\widehat{\Gamma} \widehat{S}}^{-1}\left(R^{\prime}\right)$ is homeomorphic to $\pi_{\widehat{\Gamma} \widehat{S}}^{-1}\left(x^{\prime}\right) \times R^{\prime}$. In our example, in the only non-trivial case of $R^{\prime}=\{x \in(-1,1) \mid-1 / 2<x<1 / 2\}$, we have:

$$
\pi_{\widehat{\Gamma} \widehat{S}}^{-1}\left(R^{\prime}\right)=(\widehat{S} \cap \widehat{\Gamma}) \backslash\{(1 / 2,0,1 / 2,0),(-1 / 2,0,-1 / 2,0)\}
$$

is an oval minus two points, which is homeomorphic to a Cartesian product of a pair of points $\pi_{\widehat{\Gamma} \widehat{S}}^{-1}\left(x^{\prime}\right)$ by the interval $R^{\prime}$. In other words, the connected components of $\pi_{\widehat{\Gamma} \widehat{S}}^{-1}\left(R^{\prime}\right)$ are two open arcs of simple curves.

(2) These arcs are naturally ordered separating the difference $\pi_{\widehat{\Gamma}}^{-1}\left(R^{\prime}\right) \backslash \pi_{\widehat{\Gamma} \widehat{S}}^{-1}\left(R^{\prime}\right)$ into ordered connected components. In the case of $R^{\prime}=\{x \in(-1,1) \mid-1 / 2<$ $x<1 / 2\}$ the components are (in order):

$$
\begin{aligned}
& \left\{\left(x, t, y_{1}, y_{2}\right) \in \widehat{\Gamma} \mid(-1 / 2<x<1 / 2) \wedge(f>0) \wedge\left(y_{2}<0\right)\right\}, \\
& \left\{\left(x, t, y_{1}, y_{2}\right) \in \widehat{\Gamma} \mid(-1 / 2<x<1 / 2) \wedge(f<0)\right\}, \\
& \left\{\left(x, t, y_{1}, y_{2}\right) \in \widehat{\Gamma} \mid(-1 / 2<x<1 / 2) \wedge(f>0) \wedge\left(y_{2}>0\right)\right\} .
\end{aligned}
$$

For any $x \in R^{\prime}$ the integral curve $\widehat{\Gamma}_{x}$ intersects these connected components according to their order.

(3) Each connected component of $\pi_{\widehat{\Gamma}}^{-1}\left(R^{\prime}\right) \backslash \pi_{\widehat{\Gamma} \widehat{S}}^{-1}\left(R^{\prime}\right)$ lies either in

$$
\left\{\left(x, t, y_{1}, y_{2}\right) \mid f<0\right\} \text {, }
$$

or in

$$
\left\{\left(x, t, y_{1}, y_{2}\right) \mid f>0\right\},
$$

and, therefore can be naturally labelled by $A$ or $B$ respectively. Since the connected components are ordered, the difference $\pi_{\widehat{\Gamma}}^{-1}\left(R^{\prime}\right) \backslash \pi_{\widehat{\Gamma} \widehat{S}}^{-1}\left(R^{\prime}\right)$ itself is labelled by a word (in the case of $R^{\prime}=\{x \in(-1,1) \mid-1 / 2<x<1 / 2\}$ by $B A B$ ). It follows that for any $x \in R^{\prime}$ the integral curve $\widehat{\Gamma}_{x}$ is labelled by this word, and the proposition is proved. 
Proposition 3.2 implies that the number of distinct realizable words does not exceed the number of all connected components of $R$. In our example the latter is 3 , which equals to the cardinality of the discrete set $C$ plus 1 . The general case uses a far-reaching extension of such method of counting, Alexander's duality [12].

The rest of this section is organized as follows. In Subsect. 3.2 we show how to reduce the problem of estimating the number of words realizable with respect to $\mathcal{P}$ to the similar problem with respect to a family of open subsets of $G_{2}$ such that the complement to the union of these subsets is a smooth compact hypersurface in $G_{2}$. This will allow us to describe a finite subdivision of $G_{1}$ into open subsets, within each of which the integral curves are labelled by the same word, in terms of critical points of the projection of a smooth hypersurface. This is done in Subsect. 3.3. Finally, in Subsect. 3.4, we show a single exponential upper bound on the number of all realizable words.

\subsection{Sign Sets}

Let $\mathcal{P}$ be a partition of $G_{2}=I^{n}$ into semi-Pfaffian sets. Each element of the partition is described by a Boolean combination of Pfaffian equations and inequalities defined in a domain $D$ containing the closure of $I^{n}$. Let $f_{1}, \ldots, f_{k}$ be all different functions involved in these Boolean combinations.

Definition 3.3 For a given finite set $\left\{f_{1}, \ldots, f_{k}\right\}$ of Pfaffian functions in $D$ define its sign sets as the non-empty semi-Pfaffian sets of the kind

$$
\left\{\mathbf{x} \in I^{n} \mid f_{i_{1}}=0, \ldots, f_{i_{k_{1}}}=0, f_{i_{k_{1}+1}}>0, \ldots, f_{i_{k_{2}}}>0, f_{i_{k_{2}+1}}<0, \ldots, f_{i_{k}}<0\right\},
$$

where $i_{1}, \ldots, i_{k_{1}}, \ldots, i_{k_{2}}, \ldots, i_{k}$ is a permutation of $1, \ldots, k$.

Let $\mathcal{Q}$ be the partition of $G_{2}$ into sign sets. Clearly, $\mathcal{Q}$ is a subpartition of $\mathcal{P}$, and it is sufficient to bound from above the number of words with respect to $\mathcal{Q}$.

Choose an arbitrary point in each sign set, and let $\Lambda$ be the finite set of all chosen points. There exists $\varepsilon>0$ such that for every $\mathbf{x} \in \Lambda$ and every $i, 1 \leq i \leq k$, the inequality $f_{i}(\mathbf{x})>0$ implies $f_{i}(\mathbf{x})>\varepsilon$, and $f_{i}(\mathbf{x})<0$ implies $f_{i}(\mathbf{x})<-\varepsilon$. Introduce the Pfaffian function

$$
h:=\prod_{1 \leq i \leq k}\left(f_{i}+\varepsilon\right)^{2}\left(f_{i}-\varepsilon\right)^{2} \cdot \prod_{1 \leq j \leq n}\left(1+x_{j}\right)\left(1-x_{j}\right) .
$$

It is easy to prove (see [4, Proposition 2]), that for two different sign sets $\sigma_{1}$ and $\sigma_{2}$, the singletons $\left\{\mathbf{x}_{1}\right\}=\sigma_{1} \cap \Lambda,\left\{\mathbf{x}_{2}\right\}=\sigma_{2} \cap \Lambda$ lie in different connected components of $\left\{\mathbf{x} \in I^{n} \mid h>0\right\}$ (by the choice of $\varepsilon$, neither $\mathbf{x}_{1}$ nor $\mathbf{x}_{2}$ belong to $\left\{\mathbf{x} \in I^{n} \mid h=0\right\}$ ).

Introduce the Pfaffian function $f:=h-\delta$, where $0<\delta \in \mathbb{R}$. It is easy to prove that there exists a small enough $\delta_{0}$ such that for each $\delta<\delta_{0}$ :

- the set $\left\{\mathbf{x} \in I^{n} \mid f=0\right\}$ is a smooth compact hypersurface;

- for two different sign sets $\sigma_{1}$ and $\sigma_{2}$, the singletons $\left\{\mathbf{x}_{1}\right\}=\sigma_{1} \cap \Lambda,\left\{\mathbf{x}_{2}\right\}=\sigma_{2} \cap \Lambda$ lie in different connected components of $\left\{\mathbf{x} \in I^{n} \mid f>0\right\}$. 
This defines an injective map $\varphi$ from $\mathcal{Q}$ to the set of all connected components of $\left\{\mathbf{x} \in I^{n} \mid f>0\right\}$. For each $\sigma \in \mathcal{Q}$ label the connected component $\varphi(\sigma)$ by the same letter as $\sigma$. Introduce a new letter $\chi$. Each connected component of $\left\{\mathbf{x} \in I^{n} \mid f>0\right\}$, which is not in $\varphi(\mathcal{Q})$, is labelled by $\chi$.

Definition 3.4 Define the labelling of trajectories $\Gamma_{\mathbf{x}}$ with respect to $\{f>0\}$ as follows. When $\Gamma_{\mathbf{x}}$ passes through $\varphi(\sigma)$ for some $\sigma \in \mathcal{Q}$, the letter corresponding to $\sigma$ is added to the word; when $\Gamma_{\mathbf{x}}$ passes through the connected component of $\left\{\mathbf{x} \in I^{n} \mid f>0\right\} \notin \varphi(\mathcal{Q})$, the letter $\chi$ is added to the word.

Lemma 3.5 For every word $w$, realizable with respect to $\mathcal{Q}$, there is a word $w^{\prime}$, realizable with respect to $\left\{\mathbf{x} \in I^{n} \mid f>0\right\}$, such that $w$ can be obtained from $w^{\prime}$ by deleting all occurrences of the letter $\chi$.

Proof Consider a finite set $\mathcal{G}$ of trajectories which realize all realizable words with respect to $\mathcal{Q}$. For each trajectory $\Gamma_{\mathbf{x}} \in \mathcal{G}$ and any $\sigma \in \mathcal{Q}$ choose a point in $\Gamma_{\mathbf{x}} \cap \sigma$ such that $\Gamma_{\mathbf{x}} \cap \sigma \neq \emptyset$, and let $\Lambda_{\mathbf{x}}$ be the set of all chosen points. Let $\varepsilon_{\mathbf{x}}, \delta_{\mathbf{x}}$ be the corresponding constants, and introduce $\varepsilon:=\min _{\Gamma_{\mathbf{x}} \in \mathcal{G}} \varepsilon_{\mathbf{x}}, \delta:=\min _{\Gamma_{\mathbf{x}} \in \mathcal{G}} \delta_{\mathbf{x}}$. Define the function $f$ with constants $\varepsilon, \delta$. Now the lemma follows from Definition 3.4.

Corollary 3.6 The number of words realizable with respect to $\mathcal{Q}$ does not exceed the number of words realizable with respect to $\left\{\mathbf{x} \in I^{n} \mid f>0\right\}$.

\subsection{General Case}

Let $\gamma: G_{1} \times(-1,1) \rightarrow G_{2}$, where $G_{1}=I^{n-1}$ and $G_{2}=I^{n}$, be a homeomorphism, defined by its graph

$$
\widehat{\Gamma}:=\{(\mathbf{x}, t, \mathbf{y}) \mid \gamma(\mathbf{x}, t)=\mathbf{y}\}
$$

which is a semi-Pfaffian set. Note that $\widehat{\Gamma}$ is homeomorphic to $I^{n}$.

Assume that $\widehat{\Gamma}:=\left\{(\mathbf{x}, t, \mathbf{y}) \in \mathbb{R}^{2 n} \mid F(\mathbf{x}, t, \mathbf{y})\right\}$, where $F(\mathbf{x}, t, \mathbf{y})$ is a Boolean formula in DNF with atomic Pfaffian functions $f_{i}, g_{j}$ defined in a domain containing the closure of $I^{2 n}$. Let $V$ be the singular locus of $\widehat{\Gamma}$, and $U:=\widehat{\Gamma} \backslash V$. It follows that $\operatorname{dim}(V)<n, \operatorname{dim}(U)=n$, and $U$ is a smooth $\left(C^{1-}\right)$ manifold.

For each $\mathbf{x} \in G_{1}$ consider the integral curve

$$
\widehat{\Gamma}_{\mathbf{x}}:=\{(t, \mathbf{y}) \mid \gamma(\mathbf{x}, t)=\mathbf{y}\}=\{(t, \mathbf{y}) \mid F(\mathbf{x}, t, \mathbf{y})\}
$$

and the trajectory

$$
\Gamma_{\mathbf{x}}:=\{\mathbf{y} \mid \exists t(\gamma(\mathbf{x}, t)=\mathbf{y})\}=\{\mathbf{y} \mid \exists t(F(\mathbf{x}, t, \mathbf{y}))\} .
$$

Observe that both $\widehat{\Gamma}_{\mathbf{x}}$ and $\Gamma_{\mathbf{x}}$ are homeomorphic to the interval $(-1,1)$, and that $\widehat{\Gamma}_{\mathbf{x}}$ can be naturally identified with the fiber over $\mathbf{x}$ of the projection $\pi_{\widehat{\Gamma}}: \widehat{\Gamma} \rightarrow G_{1}$.

Let $S:=\left\{\mathbf{y} \in G_{2} \mid f=0\right\} \subset G_{2}$, where $f=h-\delta$ is as defined in Subsect. 3.2. Recall that $S$ is a smooth compact hypersurface in $G_{2}$. Let the connected components of $G_{2} \backslash S=\left\{\mathbf{y} \in G_{2} \mid f^{2}>0\right\}$ be labelled by different letters of a finite alphabet. 
Then a trajectory $\Gamma_{\mathbf{x}}, \mathbf{x} \in G_{1}$, is labelled by a finite word in this alphabet (assuming the trajectory is directed). We want to estimate from above the number of distinct realizable words. Clearly, it is sufficient to consider integral curves $\widehat{\Gamma}_{\mathbf{x}}$ and smooth hypersurfaces

$$
\widehat{S}:=\left\{(\mathbf{x}, t, \mathbf{y}) \in G_{1} \times(-1,1) \times G_{2} \mid f(\mathbf{y})=0\right\} .
$$

Lemma 3.7 The intersection $U \cap \widehat{S}$ is smooth and $\operatorname{dim}(V \cap \widehat{S})<n-1$ for $\delta$ small enough.

Proof Consider the restriction $h_{U}$ of $h$ on $U$. By Sard's theorem (in o-minimal version [10]), any small enough $\delta>0$ is a regular value of $h_{U}$. Hence by the implicit function theorem the intersection $U \cap \widehat{S}$, which is the fiber of $h_{U}$ over $\delta$, is smooth.

It follows that $U \cap \widehat{S}$ is a smooth submanifold of positive codimension of both manifolds: $U$ and $\widehat{S}$. Let $L:=\pi_{\widehat{\Gamma}}(V \cap \widehat{S})$. Lemma 3.7 implies that $\operatorname{dim}(L)<n-1$.

Let $K$ be the set of all $\mathbf{x} \in G_{1}$ such that $\pi_{U}^{-1}(\mathbf{x}) \cap \widehat{S}$ contains a singular point of $\pi_{U}^{-1}(\mathbf{x})$. Then, by the implicit function theorem, $K$ is a subset of all critical values of the projection $\pi_{U}: U \rightarrow G_{1}$. It follows by Sard's theorem that $\operatorname{dim}(K)<n-1$.

Let $\pi_{U \widehat{S}}$ be the restriction of $\pi_{U}$ to $U \cap \widehat{S}$. Denote by $C$ the set of all critical values of $\pi_{U \widehat{S}}$. By Sard's theorem, $\operatorname{dim}(C)<n-1$. Observe that for any $\mathbf{x} \in C \backslash K$ the fiber $\pi_{U}^{-1}(\mathbf{x})$ is tangent to $\widehat{S}$ at some point $(\mathbf{x}, t, \mathbf{y})$. Let $R:=G_{1} \backslash(K \cup L \cup C)$. Then for any $\mathbf{x} \in R$ the fiber $\pi_{\widehat{\Gamma}}^{-1}(\mathbf{x})$ intersects $\widehat{S}$ transversally.

Proposition 3.8 If $\mathbf{x}_{1}, \mathbf{x}_{2}$ belong to the same connected component $R^{\prime}$ of $R$, then $\widehat{\Gamma}_{\mathbf{x}_{1}}$ and $\widehat{\Gamma}_{\mathbf{x}_{2}}$ are labelled by the same word.

Proof We prove the proposition in four steps.

(1) The restriction of $\pi_{U \widehat{S}}$ on $\pi_{U \widehat{S}}^{-1}\left(R^{\prime}\right)$ is proper as well as a submersion, and therefore, by the inverse function theorem (see, e.g., [7]), is a locally trivial covering. Thus, for any fixed $\mathbf{x}^{\prime} \in R^{\prime}$ the pre-image $\pi_{U \widehat{S}}^{-1}\left(W_{\mathbf{x}^{\prime}}\right)$ of a neighborhood $W_{\mathbf{x}^{\prime}}$ of $\mathbf{x}^{\prime}$ in $R^{\prime}$ is homeomorphic to $\pi_{U \widehat{S}}^{-1}\left(\mathbf{x}^{\prime}\right) \times W_{\mathbf{x}^{\prime}}$. In particular, all fibers $\pi_{U \widehat{S}}^{-1}(\mathbf{x})$ for $\mathbf{x} \in$ $W_{\mathbf{x}^{\prime}}$ are homeomorphic. It follows that each connected component of $\pi_{U \widehat{S}}^{-1}\left(W_{\mathbf{x}^{\prime}}\right)$ is diffeomorphic to $W_{\mathbf{x}^{\prime}}$, and therefore its complement in $\pi_{\widehat{\Gamma}}^{-1}\left(W_{\mathbf{x}^{\prime}}\right)$ consists of two connected components.

(2) We claim that there exists a linear order $1,2, \ldots, i, \ldots, s$ on the set of all connected components of $\pi_{U \widehat{S}}^{-1}\left(W_{\mathbf{x}^{\prime}}\right)$ such that component $i$ in that order splits $\pi_{\widehat{\Gamma}}^{-1}\left(W_{\mathbf{x}^{\prime}}\right) \backslash i$ into two connected components one of which contains components $1, \ldots, i-1$ and the other contains components $i+1, i+2, \ldots, s$. We say that $i$ separates these two sets of components. We prove the claim by induction on $s$, the base case of $s=1$ being trivial. Suppose that there is a required linear order $1,2, \ldots, s-1$ for $s-1$ components of $\pi_{U \widehat{S}}^{-1}\left(W_{\mathbf{x}^{\prime}}\right)$, where $s \geq 2$. Then the $s^{\text {th }}$ component, which we denote by $A$, either is separated from $1,2, \ldots, s-2$ by $s-1$, or is separated from $2, \ldots, s-1$ by 1 , or lies in a connected component of $\pi_{\widehat{\Gamma}}^{-1}\left(W_{\mathbf{x}^{\prime}}\right) \backslash(i \cup i+1)$ between the components $i$ and $i+1$ for some $1 \leq i \leq s-2$. In the first case label component 
$A$ by $s$, in the second case label $A$ by 1 and add 1 to the label of each the of the remaining components. In the last case notice that the smooth curve $\pi_{U}^{-1}\left(\mathbf{x}^{\prime}\right)$ intersects $i, i+1$ and $A$ transversally, and, according to (1), each intersection consists of a single point. It follows that $A$ separates components $i$ and $i+1$. Add 1 to the label of each the of the components $i+1, \ldots, s-1$ and label $A$ by $i+1$. The claim is proved.

Observe that the linear order on the set of all connected components of $\pi_{U \widehat{S}}^{-1}\left(W_{\mathbf{x}^{\prime}}\right)$ induces the linear order on the set of all connected components of $\pi_{\widehat{\Gamma}}^{-1}\left(W_{\mathbf{x}^{\prime}}\right) \backslash$ $\pi_{U \widehat{S}}^{-1}\left(W_{\mathbf{x}^{\prime}}\right)$.

(3) Each connected component of $\pi_{\widehat{\Gamma}}^{-1}\left(W_{\mathbf{x}^{\prime}}\right) \backslash \pi_{U \widehat{S}}^{-1}\left(W_{\mathbf{x}^{\prime}}\right)$ lies in a connected component of $\{f>0\}$ and therefore is labelled by a letter. In view of the linear order, the set of all connected components of $\pi_{\widehat{\Gamma}}^{-1}\left(W_{\mathbf{x}^{\prime}}\right) \backslash \pi_{U \widehat{S}}^{-1}\left(W_{\mathbf{x}^{\prime}}\right)$ is labelled by a word, say $w$. Then for any $\mathbf{x}_{1}, \mathbf{x}_{2} \in W_{\mathbf{x}^{\prime}}$ the integral curves $\widehat{\Gamma}_{\mathbf{x}_{1}}=\pi_{\widehat{\Gamma}}^{-1}\left(\mathbf{x}_{1}\right)$ and $\widehat{\Gamma}_{\mathbf{x}_{2}}=\pi_{\widehat{\Gamma}}^{-1}\left(\mathbf{x}_{2}\right)$ are labelled by $w$.

(4) Since $R^{\prime}$ is path-connected, there is a compact connected linear curve $L \subset R^{\prime}$. As we proved in (1)-(3), for each point $\mathbf{x}^{\prime} \in L$ there is an open neighborhood $W_{\mathbf{x}^{\prime}}$ of $\mathbf{x}^{\prime}$ in $R^{\prime}$ such that for any $\mathbf{x}_{1}, \mathbf{x}_{2} \in W_{\mathbf{x}^{\prime}}$ the integral curves $\widehat{\Gamma}_{\mathbf{x}_{1}}, \widehat{\Gamma}_{\mathbf{x}_{2}}$ are labelled by the same word $w$. By compactness of $L$, there is a finite family of open sets $W_{\mathbf{x}^{\prime}} \cap L$ in $L$ which is a covering of $L$. It follows that if now $\mathbf{x}_{1}, \mathbf{x}_{2} \in R^{\prime}$, then $\widehat{\Gamma}_{\mathbf{x}_{1}}$ and $\widehat{\Gamma}_{\mathbf{x}_{2}}$ are labelled by $w$.

\subsection{Upper Bound}

Proposition 3.8 implies that to bound from above the number of all realizable words we need to estimate the number of connected components of $R=G_{1} \backslash(K \cup L \cup C)$.

We first write out an existential formula $\mathcal{L}(\mathbf{x})$ for $L$ using Theorem 2.22. Then we construct an existential formula for $K \cup C$. For each $\mathbf{x} \in G_{1} \backslash L$ introduce the secant bundle

$$
\mathcal{S} \widehat{\Gamma}_{\mathbf{x}}:=\left\{(t, \mathbf{y}, \mathbf{v}) \in \widehat{\Gamma}_{\mathbf{x}} \times \mathbb{R}^{n+1} \mid(t, \mathbf{y}, t, \mathbf{y}, \mathbf{v}) \in \operatorname{closure}(\mathcal{S})\right\}
$$

where

$$
\begin{aligned}
\mathcal{S}:= & \left\{\left(\mathbf{x}, t, \mathbf{y}, t^{\prime}, \mathbf{y}^{\prime}, \lambda\left((t, \mathbf{y})-\left(t^{\prime}, \mathbf{y}^{\prime}\right)\right) \in \widehat{\Gamma}_{\mathbf{x}} \times \widehat{\Gamma}_{\mathbf{x}} \times \mathbb{R}^{n+1} \mid\right.\right. \\
& \left.\left.\left((t, \mathbf{y}) \neq\left(t^{\prime}, \mathbf{y}^{\prime}\right)\right) \wedge(\lambda \in \mathbb{R})\right)\right\} .
\end{aligned}
$$

According to Lemma 2.19, for $\mathbf{x} \in G_{1} \backslash L$ the singular points of $\widehat{\Gamma}_{\mathbf{x}}$ belonging to $\widehat{S}$ are defined by the formula

$$
\mathcal{K}(\mathbf{x}):=\exists \mathbf{z}_{1}, \mathbf{z}_{2}\left(\mathbf{z}_{1} \in \mathcal{S}_{(t, \mathbf{y})} \wedge \mathbf{z}_{2} \in \mathcal{S}_{(t, \mathbf{y})} \wedge \operatorname{rank}\left(\mathbf{z}_{1}, \mathbf{z}_{2}\right)=2 \wedge f(\mathbf{y})=0\right) .
$$

Hence, $K \cup L=\{\mathbf{x} \mid \mathcal{K}(\mathbf{x}) \vee \mathcal{L}(\mathbf{x})\}$.

Observe that for $\mathbf{x} \in G_{1} \backslash(L \cup K)$ a point $(t, \mathbf{y})$ is a tangent point of $\widehat{\Gamma}_{\mathbf{x}}$ to $\widehat{S}$ iff

$$
\exists \mathbf{v} \in \mathbb{R}^{n+1}\left(\left((t, \mathbf{y}, \mathbf{v}) \in \mathcal{S} \widehat{\Gamma}_{\mathbf{x}}\right) \wedge\left(\left\langle\mathbf{v}, \operatorname{grad}_{(t, \mathbf{y})} \widehat{S}\right\rangle=0\right) \wedge\left((t, \mathbf{y}) \in \widehat{\Gamma}_{\mathbf{x}} \cap \widehat{S}\right)\right) .
$$


Denoting the latter formula by $\mathcal{C}$, we get

$$
K \cup L \cup C=\left\{\mathbf{x} \in G_{1} \mid \mathcal{K}(\mathbf{x}) \vee \mathcal{L}(\mathbf{x}) \vee \mathcal{C}(\mathbf{x})\right\}
$$

thus $K \cup L \cup C$ is defined by an existential formula.

If functions in formula $F(\mathbf{x}, t, \mathbf{y})$ and the function $f$ are Pfaffian, then, according to Theorem 2.18, the bound on the Betti number $\mathrm{b}_{n-1}(K \cup L \cup C)$ is expressible via the format of $\mathcal{K}(\mathbf{x}) \vee \mathcal{L}(\mathbf{x}) \vee \mathcal{C}(\mathbf{x})$. By Alexander's duality $([6$, Lemmas 4, 5] and [12]), the number of connected components of $G_{1} \backslash(K \cup L \cup C)$,

$$
\mathrm{b}_{0}\left(G_{1} \backslash(K \cup L \cup C)\right) \leq \mathrm{b}_{n-1}(K \cup L \cup C) .
$$

Assume that formula $F(\mathbf{x}, t, \mathbf{y})$ and the formula defining partition $\mathcal{P}$ (as the union of elements of the partition) both have format $(r, N, \alpha, \beta, 2 n)$. According to Theorem 2.22, the sub-Pfaffian set $K \cup L \cup C$ has the format $\left(r,\left(N^{O(n)} D\right)^{n+r}, \alpha, D\right.$, $\left.O\left(n^{2}\right)\right)$, where

$$
D:=2^{O\left(n r^{2}\right)}(n(\alpha+\beta))^{O(n(n+r))} .
$$

Then, by Theorem 2.18,

$$
\mathrm{b}_{n-1}(K \cup L \cup C) \leq N^{O\left(n^{4}\right)} 2^{O\left(n^{4} r^{2}(n+r)\right)}(n(\alpha+\beta))^{O\left(n^{4}(n+r)^{2}\right)},
$$

which is also the upper bound on $|\Omega|$. The cardinality of the set $\dot{\Omega}$ of all dotted words does not exceed $|\Omega|$ multiplied by the upper bound on the length $\ell$ of a word $\mathbf{x} \in G_{1}$. The latter equals to the number of the connected components of the intersection $\widehat{\Gamma}_{\mathbf{x}} \cap(\widehat{\Gamma} \backslash \widehat{S})$. By Theorem 2.17,

$$
\ell \leq N^{O(n)} 2^{r^{2}}(n(\alpha+\beta))^{O(n+r)} .
$$

It follows that $|\dot{\Omega}|=|\Omega| \ell$ is bounded from above by the right-hand side of (3.3).

We proved the following theorem.

Theorem 3.9 Let $T_{\gamma}=\left(G_{2}, \rightarrow\right)$ be the transition system associated to the dynamical system $\gamma$. Then there is a bisimulation on $T_{\gamma}$ with respect to $\mathcal{P}$ consisting of at most

$$
N^{O\left(n^{4}\right)} 2^{O\left(n^{4} r^{2}(n+r)\right)}(n(\alpha+\beta)){ }^{O\left(n^{4}(n+r)^{2}\right)}
$$

equivalence classes, where $D$ is defined in (3.2).

Relaxing the bound from Theorem 3.9, we get that the number of equivalence classes in a finite bisimulation does not exceed

$$
N^{O\left(n^{4}\right)}(n(\alpha+\beta))^{O\left(n^{6} r^{3}\right)} .
$$

Remark 3.10 The best upper bound known until now ([8]) was double exponential:

$$
N^{(r+n)^{O(n)}}(\alpha+\beta)^{(r+n)^{O\left(n^{3}\right)}} .
$$




\section{Lower Bound}

We construct a parametric example of a semi-algebraic dynamical system $G_{1} \times$ $(-1,1) \rightarrow G_{2}$ together with a semi-algebraic partition of $G_{2}$ such that the format of both of them is $(d, n)$ (degrees, number of variables) while the number of different realizable words (size of a bisimulation) is $d^{\Omega(n)}$.

Let $g(y)$ be a polynomial of degree $d$ such that $|g(y)|<1$ for every $y \in(-1,1)$ and for every $c \in\left(-\frac{1}{2}, \frac{1}{2}\right)$ the polynomial $g(y)-c$ has $d$ simple roots in $(-1,1)$.

First we illustrate the idea of the example by describing the case $n=2$. Let the dynamical system be given by $G_{1}:=(-1,1), G_{2}:=(-1,1)^{2}, \gamma:(\mathbf{x}, t) \mapsto(t, \mathbf{x})$. The partition $\mathcal{P}$ consists of two sets $A$ and $B=G_{2} \backslash A$ where

$$
A:=\left\{\left(y_{1}, y_{2}\right) \mid g\left(y_{1}\right)=0, y_{1}+y_{2}>0\right\} .
$$

Notice that there are exactly $d+1$ distinct words encoding all trajectories of the defined dynamical system. These words are formed by alternating letters starting and ending with $B$, i.e., $B, B A B, B A B A B, \ldots$

For arbitrary $n$, let $G_{1}:=(-1,1), G_{2}:=(-1,1)^{n}$. Define a curve

$$
\Delta:=\left\{\left(y_{1}, \ldots, y_{n-1}\right) \in(-1,1)^{n-1} \mid y_{2}=g\left(y_{1}\right), \ldots, y_{n-1}=g\left(y_{n-2}\right)\right\} .
$$

Observe that $\Delta$ is connected in $(-1,1)^{n-1}$, being the graph of the map

$$
\begin{gathered}
\mathbf{f}:(-1,1) \rightarrow(-1,1)^{n-1}, \\
y_{1} \mapsto\left(g\left(y_{1}\right), \ldots, g\left(g\left(\cdots g\left(y_{1}\right) \cdots\right)\right)\right),
\end{gathered}
$$

and smooth.

Consider the polynomial $h\left(y_{n-1}\right):=\left(y_{n-1}-b_{1}\right)\left(y_{n-1}-b_{2}\right) \cdots\left(y_{n-1}-b_{d}\right)$ where all $b_{i} \in\left(-\frac{1}{2}, \frac{1}{2}\right)$ and $b_{i} \neq b_{j}$ for $i \neq j$. Then $\Delta \cap\{h=0\}$ consists of $d^{n-1}$ points. Define

$$
A:=\left\{\left(y_{1}, \ldots, y_{n}\right) \mid\left(y_{1}, \ldots, y_{n-1}\right) \in \Delta, h\left(y_{n-1}\right)=0, L>0\right\},
$$

where $L\left(y_{1}, \ldots, y_{n}\right)$ is a generic linear homogeneous polynomial such that $\{L=0\}$ intersects all $d^{n-1}$ parallel straight lines of

$$
\left\{\left(y_{1}, \ldots, y_{n}\right) \mid\left(y_{1}, \ldots, y_{n-1}\right) \in \Delta, h\left(y_{n-1}\right)=0\right\} .
$$

Notice that the projection of this intersection on the $y_{n}$-coordinate consists of $d^{n-1}$ distinct points.

Finally, define the dynamical system $\gamma$ and the partition $\mathcal{P}$ as follows. To $\mathbf{x} \in G_{1}$ and $t \in(-1,1)$ the map $\gamma$ assigns the point $(\mathbf{f}(t), \mathbf{x}) \in G_{2}$. The partition $\mathcal{P}$ consists of $A$ and $B=G_{2} \backslash A$. Clearly, there are exactly $d^{n-1}+1$ pairwise distinct words encoding all trajectories.

We can now modify the example so that $G_{1}$ becomes homeomorphic to $I^{n-1}$, and $G_{2}$ becomes homeomorphic to $I^{n}$. Observe that there is a small enough $\varepsilon>0$ such 
that for any sequence $0<\varepsilon_{1}, \ldots, \varepsilon_{n-2} \leq \varepsilon$ and any sequence $*_{1}, \ldots, *_{n-2} \in\{+,-\}$, the algebraic set

$$
\Delta^{\prime}:=\left\{\left(y_{1}, \ldots, y_{n-1}\right) \in(-1,1)^{n-1} \mid y_{2}=g\left(y_{1}\right) *_{1} \varepsilon_{1}, \ldots, y_{n-1}=g\left(y_{n-2}\right) *_{n-2} \varepsilon_{n-2}\right\}
$$

is a smooth connected curve. These curves are disjoint and their union is

$$
\Delta^{\prime \prime}:=\bigcap_{1 \leq i \leq n-2}\left\{\left(y_{1}, \ldots, y_{n-1}\right) \in(-1,1)^{n-1} \mid-\varepsilon<y_{i+1}-g\left(y_{i}\right)<\varepsilon\right\} .
$$

Let $G_{1}:=(-\varepsilon, \varepsilon)^{n-2} \times(-1,1), G_{2}=\Delta^{\prime \prime} \times(-1,1)$ and $\gamma: G_{1} \times(-1,1) \rightarrow G_{2}$, such that

$$
\left(*_{1} \varepsilon_{1}, \ldots, *_{n-2} \varepsilon_{n-2}, x, t\right) \mapsto\left(g(t) *_{1} \varepsilon_{1}, \ldots, g(g(\cdots g(t) \cdots)) *_{n-2} \varepsilon_{n-2}, x\right) .
$$

Note that $\gamma$ is a diffeomorphism. It is obvious that the modified $\gamma$ still has at least $d^{\Omega(n)}$ trajectories with pairwise distinct word codes with respect to the partition $\mathcal{P}$.

\section{Future Work}

In [8] the authors proposed an algorithm (a Blum-Schub-Smale type machine with an oracle for deciding non-emptiness of semi-Pfaffian sets) for computing a finite bisimulation. That algorithm is based on the cylindrical cell decomposition technique and, accordingly, has a double exponential upper complexity bound. It seems feasible to construct a bisimulation algorithm with single exponential complexity using the approach employed in the present paper. Once a bisimulation is computed, it can be used in efficient algorithms for fundamental computational problems such as deciding reachability or motion planning in definable dynamical systems.

Acknowledgements The authors would like to thank A. Gabrielov, V. Grandjean, K. Korovin for useful discussions, and the anonymous referee for valuable suggestions.

The first author was partly supported by Grant Scientific School-4413.2006.1, DFG-RFBR 436 RUS 06/01/04. The second author was supported in part by the European RTN Network RAAG (contract HPRNCT-2001-00271).

\section{References}

1. Basu, S., Pollack, R., Roy, M.-F.: Algorithms in Real Algebraic Geometry. Springer, Berlin (2003)

2. Brihaye, T., Michaux, C., Riviere, C., Troestler, C.: On o-minimal hybrid systems. In: Alur, R., Pappas, G.J. (eds.) Proceedings of HSCC 2004. Lecture Notes in Comput. Sci., vol. 2993, pp. 219-233 (2004)

3. Davoren, J.M.: Topologies, continuity and bisimulations. Theor. Inf. Appl. 33(4/5), 357-382 (1999)

4. Gabrielov, A., Vorobjov, N.: Complexity of stratifications of semi-Pfaffian sets. Discret. Comput. Geom. 14, 71-91 (1995)

5. Gabrielov, A., Vorobjov, N.: Complexity of computations with Pfaffian and Noetherian functions. In: Ilyashenko, Y. et al. (eds.) Normal Forms, Bifurcations and Finiteness Problems in Differential Equations. NATO Science Series II, vol. 137, pp. 211-250. Kluwer, Dordrecht (2004)

6. Gabrielov, A., Vorobjov, N., Zell, T.: Betti numbers of semialgebraic and sub-Pfaffian sets. J. Lond. Math. Soc. 69(1), 27-43 (2004) 
7. Hirsch, M.: Differential Topology. Springer, New York (1976)

8. Korovina, M., Vorobjov, N.: Pfaffian hybrid systems. In: Computer Science Logic'04. Lecture Notes in Comput. Sci., vol. 3210, pp. 430-441 (2004)

9. Korovina, M., Vorobjov, N.: Upper and lower bounds on sizes of finite bisimulations of Pfaffian hybrid systems. In: Logical Approaches to Computational Barriers. Lecture Notes in Comput. Sci., vol. 3988, pp. 267-276

10. Kurdyka, K., Orro, P., Symon, S.: Semialgebraic Sard theorem for generalized critical values. J. Differ. Geom. 56, 67-92 (2000)

11. Lafferriere, G., Pappas, G., Sastry, S.: O-minimal hybrid systems. Math. Control Signals Syst. 13, $1-21(2000)$

12. Massey, W.S.: A Basic Course in Algebraic Topology. Springer, New York (1991)

13. Zell, T.: Betti numbers of semi-Pfaffian sets. J. Pure Appl. Algebra 139, 323-338 (1999) 